\title{
Generic Adaptive Moving Object Tracking Algorithms
}

\author{
Jing Zhou ${ }^{\dagger} \quad$ Hong Va Leong ${ }^{\dagger}$ \\ ${ }^{\dagger}$ Department of Computing \\ The Hong Kong Polytechnic University \\ Hong Kong \\ \{csjgzhou, cshleong, csluqin\}@ comp.polyu.edu.hk \\ Qin $\mathrm{Lu}^{\dagger} \quad$ Ken C.K. Lee \\ ${ }^{\ddagger}$ Dept. of Computing Science and Engr. \\ Pennsylvania State University \\ University Park, PA 16802 \\ cklee@cse.psu.edu
}

\begin{abstract}
Moving Object Databases (MODs), the core component of location server to support location-related applications, keep track of the locations of moving objects which submit location update reports to the centralized server. In resource-limited wireless environments, the frequency and conditions for generating location update messages exert a strong impact on system performance in terms of update message cost and object location accuracy, hence the query result precision. Conceptually, moving objects are the sources of the location data while the MOD caches recently reported object locations for query processing. Owing to the inherent imprecision of the cached values, we impose a bounded level of inconsistency for the cached values, realized in the form of a "safe range" for a moving object. The cached value needs not be invalidated so long as the deviation of the object's current location from its reported location is within the safe range. A smaller safe range results in a higher accuracy of the cached value and hence more accurate query result at the expense of higher update cost, and vice versa. Since the size of the safe range is the key to system performance, we derive a system cost model to determine its appropriate value. Furthermore, to cater for highly dynamic environments in which object movement, query access pattern and system workload always change, we propose two adaptive safe range adjustment algorithms. Through extensive simulation experiments, the benefits brought about by our algorithms are evidenced.
\end{abstract}

\section{Introduction}

Moving object location tracking is an essential service in many location-related applications such as intelligent transportation system, logistics, fleet/cargo management, childcare system, wildlife animal monitoring, emerging service like E911 etc. Consider the intelligent transportation system, often launched in a city for answering queries from drivers, passengers, police and other interested parties. One typical task is to keep monitoring locations of interested moving objects and log the updated location value to a Moving Object Database (MOD) for centralized efficient query processing. Figure 1 depicts a MOD, the core component of location server, keeping track of the locations of a large number of moving objects such as persons, vehicles, and wildlife animals and processing user queries on these moving objects. Under this configuration, moving objects report their locations to the MOD either periodically, on demand, or when a certain condition materializes. Maintaining reported locations, MOD generates results to user queries about the locations of interested moving objects.

In our model, the system performance is attributed to two costs: location update cost and query evaluation cost. The location update cost is basically the cost to post location updates from battery-powered moving objects to the database server over a scare-bandwidth wireless channel. Clearly, it is impractical for an object to keep submitting location reports continuously along its trajectory. To reduce the update cost, state-of-art update policies like time-based [1], distance-based [11], dead-reckoning [14], adaptive deadreckoning [7] and group-based location update [6] have been proposed. The query evaluation cost is resulted when the object location at server is imprecise and the server needs to page the object for its updated location.

Conceptually, the moving objects are the sources of their location information (source data) and the database server maintains a cache of latest reported object locations (cached data). The problem of location update and querying resembles the cache coherence problem. Though location update can be realized as cache update or cache invalidation initiated by moving objects to MOD, we adopt cache update approach since the payload of a location update message that includes object ID and spatial coordinates is very close to an invalidation message that contains the object ID only.

In this paper, we propose a distance-based update approach with adaptively tuned safe range to realize the concept of location cache at MOD. The safe range defines the degree of bounded inconsistency [16], which is the maximum deviation allowed between the cached location and the actual location. Thus, when an object moves to a new position within the safe range, the cached value remains valid; no update needs to be issued to the server. From the per- 
cached location within the safe range, in the absence of an update. The object location may be stale, but accurate enough to answer queries with fair accuracy expectation. For queries with high accuracy requirement, relevant objects will be paged when queried. This on-demand paging cost, resulted when high accuracy queries access object location with low precision, constitutes the query evaluation cost. This object paging resembles cache verification.

In our algorithms, the adaptation of safe ranges is mutually agreed between the server and the moving objects. Based on the safe range $r$, a location update is initiated only when the object is more than a distance of $r$ away from its previously reported location. The area within which no update is needed is called the safe region. It is a circular area centered at the previously reported location with radius $r$.

Although to a certain degree, the precision of cached value is bounded, processing a query with a high precision requirement, as specified by users, could likely trigger object paging. We should set the safe range judicially to improve system performance. However, selecting a good safe range needs thorough analysis since a small safe range is not effective in reducing location update frequency, while a large safe range incurs high object paging cost, especially when a large volume of queries are to be served, despite suppressing the location updates. Even more complicated, the safe range is not necessarily fixed; it should be adaptive in highly dynamic environments in which object movement pattern and query access pattern always change. To address this, we propose two adaptive safe range determination algorithms that are both movement-aware and queryaware [18]. To evaluate, we conduct extensive experiments with synthetic datasets. The experimental results well indicate their effectiveness in balancing the update cost and query cost, resulting in an optimal system performance.

The rest of this paper is organized as follows. Section 2 reviews the related work. Section 3 describes the general system model and the operation of each component. Section 4 derives the system cost model, providing the basis to develop our adaptive algorithms discussed in Section 5. We conduct experiments to evaluate the performance of our adaptive algorithms, demonstrating their effectiveness in yielding minimal operational cost. Details are documented in Section 6. Finally, Section 7 summarizes our work and highlights some future research directions.

\section{Related Work}

In distributed information systems, the problem of adaptive data caching is related to adjusting the caching strategy dynamically as conditions change $[3,4,13]$. Most previous work does not focus on the problem of how to set cache precision optimally according to data value change and query situation [2, 10, 12, 17]. A distinguished work addressing the optimization problem considers interval approximation ing the precision of cached approximations [9]. One of our algorithms extends this methodology and makes the general approach applicable for MOD applications.

Although moving object tracking is essential in mobile computing applications, little work is done in MOD to address the location information caching optimization problem. A handful of tracking algorithms and updating policies such as time-based, distance-based, dead-reckoning are proposed $[1,7,11,14]$ and comparison work has been done [11]. However, none of the existing work focuses on optimization efforts. To our best knowledge, the most relevant work taking the optimization approach is from Wolfson [14]. In their work, an information cost model is proposed based on three separate costs: update cost, deviation cost and uncertainty cost. They derive the optimal settings for deviation threshold to yield the minimum information cost, based on the dead-reckoning policies. In their model, queries are not allowed to request for exact values from sources so server probe costs are not taken into account. Compared to their work, ours is much more adaptive in the sense that it is both query-aware and movement-aware [18].

The novelty of our approach lies in three aspects. First, Wolfson's work regards querying and updating as two separate procedures in MOD environment, in which the uncertainty and deviation impose a cost or penalty in terms of incorrect decision making. Based on this, the information cost function is designed to absorb both update cost and penalty for uncertainty, so as to derive the minimal cost for one trip by assigning the optimal value to the object's deviation threshold. They then consider the query issued in a next step. We believe that in most practical systems, the only reason to provide up-to-date location information is to provide answers precise enough to queries concerning these objects. Therefore, if no query is issued for the whole trip of a moving object, it is not necessary to "produce" the information for the non-existent "consumer". Thus, both movement pattern and query information are absorbed and integrated into our adaptive algorithms. Second, the dead-reckoning policies suffer from the dependency of the optimal deviation threshold value on a predicted deviation function. The deviation threshold at each update is adjusted to the current motion pattern, whose changes need to be reflected by parameter change on the predicted deviation function, such as the linear function, $d(t)$ [14]. In contrast, our adaptive algorithms do not need to be based on any predicted deviation function. Third, the dead-reckoning policies are only applicable when the destination and motion plan of the moving objects are known a priori [15]. The route would be fixed and known to both the moving object and the server. However the future route of a tracked object is not always known. Our work relaxes the assumption about predefined routes or destination about the motion plan. 
Figure 1 depicts the general updating and querying activities in the MOD, which resides inside some kind of location server providing location-dependent querying services. The location server communicates with moving objects via a low bandwidth network and records their locations. Upon receiving queries issued by users, location server processes them and returns the results. It is normally the responsibility of the moving objects to generate update reports on their locations to the server. Both server and moving objects are aware of the update policies and the agreeed safe range of location information stored in the MOD.

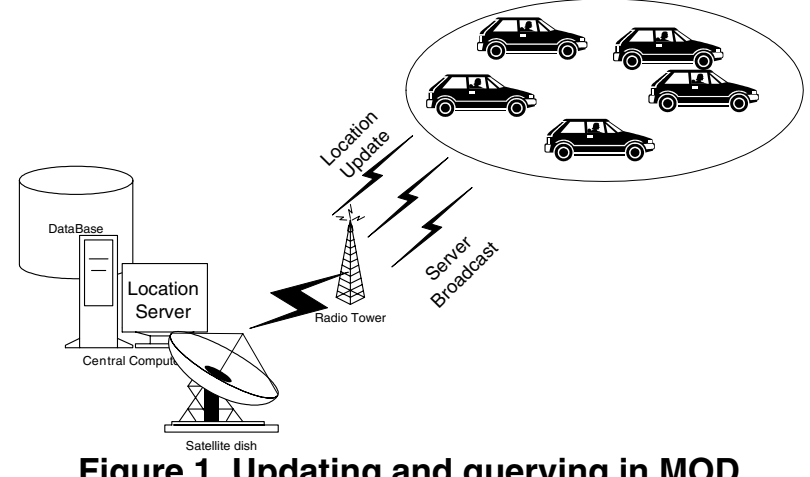

Figure 1. Updating and querying in MOD

Let the set of moving objects be $\mathcal{O}=\left\{o_{1}, o_{2}, o_{3}, \ldots\right\}$ and the set of queries issued be $\mathcal{Q}=\left\{q_{1}, q_{2}, q_{3}, \ldots\right\}$. Our adaptive algorithms are built based on the distance-based update policy. A moving object, $o \in \mathcal{O}$, issues an update whenever the distance between the current object location, $o . \operatorname{loc}_{\text {current }}$, and the stored location, $o . l c_{\text {stored }}$, exceeds the safe range $r$, i.e., $|o.| \mathrm{loc}_{\text {current }}, o .\left|\mathrm{loc}_{\text {stored }}\right|>r$. Upon each update, a tuple of the current location and chosen safe range $r_{i}$ for each object $o_{i}$ is stored at the server. Queries processed at the server are of the form $q_{j}=\left\langle o_{i}, p_{j}\right\rangle$, where $o_{i}$ is the identification for the required tracking object, and $p_{j}$ is the precision constraint for query $q_{j} \in \mathcal{Q}$. In general, to process $q_{j}$, if $r_{i} \leq p_{j}$, the stored location at the server satisfies the precision requirement and is returned to the query issuer immediately. If $r_{i}>p_{j}$, the stored location is inadequate in precision. The server needs to page the moving object $o_{i}$ for its current location, $o_{i} \cdot$ loc $_{\text {current }}$.

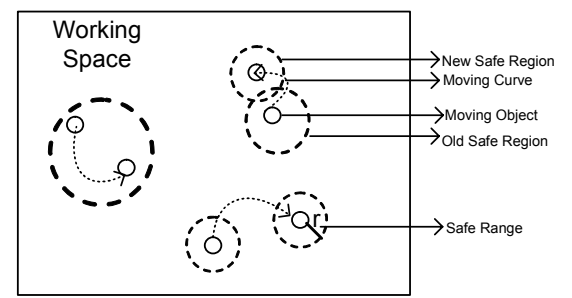

Figure 2. Moving object safe region

The procedures executed at a moving object $o_{i}$ and the server $S$ in order to maintain the MOD and to process the queries are illustrated in Figures 3 and 4. There is a safe within which there is no need for the object to report its location. An update is only needed when the object moves out of its safe region. The safe region is a circle centered at the previously reported location (the stored location at the server), with radius $r_{i}$. It is represented by the dotted circle in Figure 2 for each object. In other words, an object reports its new location if the deviation in location exceeds the safe range. Upon receiving a location update, the server installs the new location, determines the new safe range and informs the moving object. Meanwhile, the server monitors querying activities to adjust the safe range. The new safe range $r_{i}$ for $o_{i}$ is determined based on our adaptive algorithms discussed in Section 5.

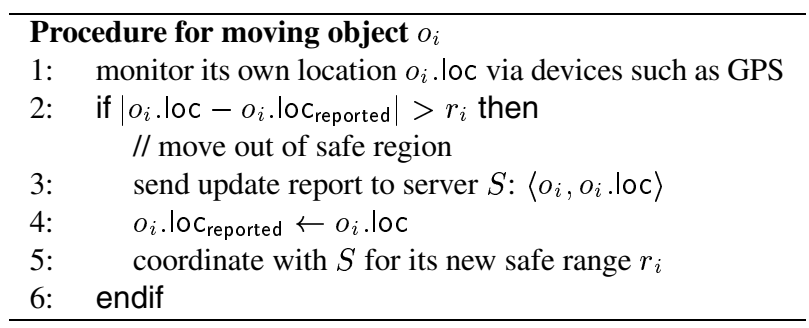

\section{Figure 3. Moving object responsibility}

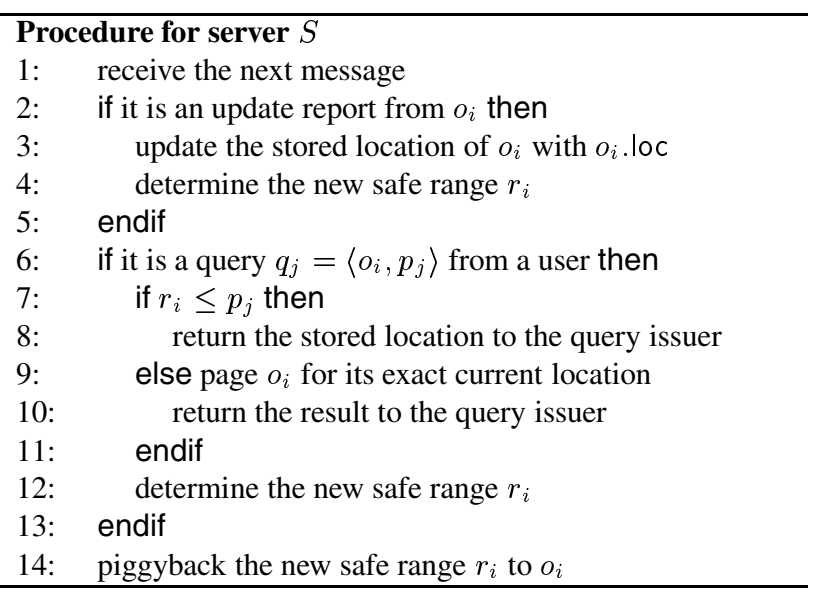

Figure 4. Server responsibility

\section{Cost-based Analysis}

The two kinds of costs, namely, wireless communication cost for location update and the query evaluation cost at the database server, are the most important factors on the system performance. Both are directly related to the location safe range, i.e., the value of the threshold $r$ to generate an update report. In order to optimize the system performance, we adopt the cost-based approach in analyzing the performance, striking at the minimal cost with an optimal setting for $r$. The total system cost, $\mathcal{C}$, can be considered as a sum of the two component costs, $C^{T}$ and $C^{Q}$, where $C^{T}$ represents the tracking cost and $C^{Q}$ stands for the querying cost. Thus, $\mathcal{C}=C^{T}+C^{Q}$. This cost function definition sets up the ultimate goal for our adaptive algorithms: minimization 
key factors that are influential to each of the costs and derive the appropriate settings to effect our adaptive algorithms design in optimizing the total $\operatorname{cost} \mathcal{C}$.

\subsection{Tracking Cost for Moving Objects}

We assume in this paper that the tracking of moving objects is achieved by voluntarily issuing update reports from moving objects to the server. Cost of sending updates upon a paging request from the server belongs to probing cost and will be associated with the query instead, as discussed in next section. The cost for tracking mainly depends on the cost for update activities and we define the tracking cost, $C_{i}^{T}$, for an object $o_{i}$ as:

$$
C_{i}^{T}=C_{u} \rho\left(r_{i}\right)
$$

where $C_{u}$ is the unit cost for an update activity. In other words, it is the cost paid for one object to send one update report to the server. The value of $C_{u}$ is applicationdependent. $\rho\left(r_{i}\right)$ is update rate for moving object $o_{i}$, i.e., the number of updates initiated from $o_{i}$ per time unit, with safe range $r_{i}$. Intuitively, larger $r_{i}$ leads to smaller rate. Hereafter, we will drop the subscript when it is clear from the context. Obviously, $\rho(r)$ depends on the movement pattern of the objects.

To define the formula for $\rho(r)$, we need to analyze the object movement behavior. Let us assume that the moving objects obey a 2-dimensional random walk model. All the objects move in steps and in each step, each object travels a distance of $d$ along an arbitrary direction. Each step takes a duration of $L$ time units.

Lemma 1 If the movement of an object $o_{i}$ follows the random walk model, each movement step lasting for a period of time $L_{i}$ and the distance moved in each step being $d_{i}$, then the rate at which $o_{i}$ moves out of the safe region is $d_{i}^{2} /\left(L_{i} r_{i}^{2}\right)$.

Proof. To prove this lemma, let us review the wellknown Drunken Person Problem.

Drunken Person Problem. A drunken person moves following the random walk model. Suppose that in every step, she moves a unit distance. After $n$ steps, the distance between her current location and the starting point is $\sqrt{n}$.

The movement of an object is similar to the drunken person. The lemma can be proved with result from the Drunken Person Problem. For an object $o_{i}$ to move out of its safe region, it should move at least a distance of $r$ beyond its safe region center, corresponding to the starting point in the drunken person problem. The normalized distance from the starting point to the boundary is $\frac{r_{i}}{d_{i}}$. Let $T$ be the expected time that $o_{i}$ moves out of its safe region. It is obvious that $\rho\left(r_{i}\right)=\frac{1}{T}$. Suppose at $t_{0}, o_{i}$ is located at safe region center and at time $t_{0}+T$, it is expected to distance moved between this time period is $D_{1}=\frac{r_{i}}{d_{i}}$ and the number steps that $o_{i}$ moves is $n=\frac{T}{L_{i}}$. Thus, the distance between the starting point and the current point is $D_{2}=\sqrt{n}=\sqrt{\frac{T}{L_{i}}}=\sqrt{\frac{1}{L_{i} \rho\left(r_{i}\right)}}$. Since $D_{1}=D_{2}$, we have $\frac{r_{i}}{d_{i}}=\sqrt{\frac{1}{L_{i} \rho\left(r_{i}\right)}}$. Thus, $\rho\left(r_{i}\right)=\frac{d_{i}^{2}}{L_{i} r_{i}^{2}}$.

Similar to [9], we can generalize the relationship between $\rho(r)$ and $r$ as:

$$
\rho(r)=\alpha / r^{2}
$$

where $\alpha$ is a parameter that represents other factors except $r$ that will affect the value of $\rho(r)$. By intuition, we know that $\alpha$ depends on the movement pattern of the objects.

\subsection{Querying Cost for Probing Moving Objects}

Besides object tracking cost, the other cost for MOD is the query processing cost. Assuming negligible CPU cost, the query processing cost is attributed to unsatisfactory safe range in location information for queries with high precision constraint. The server then needs to probe for more precise location of the moving objects involved. The costs of probing and updating from those objects comprise the querying cost, $C^{Q}$. We define the querying $\operatorname{cost} C_{j i}^{Q}$ involving an object $o_{i}$ to answer a specific query $q_{j}$ as:

$$
C_{j i}^{Q}=C_{p} \phi\left(r_{i}\right)
$$

where $C_{p}$ is unit cost for probing moving objects for the current location, which translates into the cost paid to page one object and receiving the reply back. $\phi\left(r_{i}\right)$ is query probe rate for moving object $o_{i}$, i.e., the number of probes generated per time unit, when the query $q_{j}$ is not satisfied with an object $o_{i}$ with safe range $r_{i}$, for which the query precision constraint is not fulfilled. Obviously, $\phi(r)$ depends on querying pattern, including query arrival rate and precision requirement. Intuitively, with a larger value of $r$, higher probe rate will be resulted.

To define function $\phi(r)$, we assume a simplified case where queries arrive with rate $\lambda$, each being accompanied by a precision constraint sampled from a uniform distribution, $\mathcal{U}\left(0, p_{\max }\right)$. Then $\phi(r)$ is the number of queries issued per time unit multiplied by the probability that the precision constraint of the query is not satisfied. The probability that the precision constraint of the query is not satisfied can be computed as $\operatorname{Pr}(p<r)=r / p_{\max }$. We can evaluate the function $\phi(r)$ for an object with safe range $r$ as $\phi(r)=\lambda r / p_{\max }$. We can then generalize the relationship between $r$ and $\phi(r)$ as:

$$
\phi(r)=\beta r
$$

where $\beta$ is a summarizing factor other than $r$ that could affect $\phi(r)$. Obviously, $\beta$ depends on the distribution of query precision constraint and query arrival pattern. 
With $\phi(r)$ and $\rho(r)$ determined, we can define a complete general cost function for moving objects. Table 1 summarizes all the parameters we use in the cost function and in our simulation study. We would relax the assumption in the derivation of the functions in our performance study to illustrate the robustness of our algorithms.

\begin{tabular}{|c|l|}
\hline Symbol & \multicolumn{1}{c|}{ Description } \\
\hline $\mathcal{C}$ & Total cost for the system \\
\hline$C^{T}, C^{Q}$ & Tracking and querying cost \\
\hline$C_{u}, C_{p}$ & Unit cost for location update and probe \\
\hline$r, r^{*}$ & Safe range and its optimal value \\
\hline$\rho(r), \phi(r)$ & Object update and query probe rate \\
\hline$\alpha$ & Parameter due to object movement behavior \\
\hline$\beta$ & Parameter due to querying behavior \\
\hline$\delta$ & Cost ratio, defined as $\frac{\phi(r)}{\rho(r)}$ \\
\hline$\delta^{*}$ & Optimal cost ratio, $2 \frac{C_{u}}{C_{p}}$ \\
\hline$\tau$ & Rate of adaptation \\
\hline$\omega$ & Exponential aging parameter \\
\hline$\epsilon$ & Ping-pong effect barrier parameter \\
\hline
\end{tabular}

Table 1. Symbols and parameters

Recall that $\mathcal{C}=C^{T}+C^{Q}=C_{u} \rho(r)+C_{p} \phi(r)$. With appropriate parameter values of $\alpha$ and $\beta, \mathcal{C}=\alpha / r^{2}+\beta r$. Through mathematical analysis, we can derive the minimal value for the total cost via differentiation. This occurs when $r^{*}=2 C_{u}(\sqrt[3]{\alpha / \beta}) / C_{p}$

For example, if the moving object follows the random walk model, queries are generated with a stable arrival rate of $\lambda$, and precision constraints are uniformly distributed within 0 to $p_{\max }$, We can derive $\alpha$ and $\beta$ as $\alpha=d^{2} / L$ and $\beta=\lambda / p_{\max }$. We can then easily achieve the optimal cost by setting the safe range to $r^{*}=2\left(C_{u} / C_{p}\right)\left(\sqrt[3]{d^{2} p_{\max } / \lambda L}\right)$.

Unfortunately, setting the safe range $r$ to the optimal $r^{*}$ is difficult unless object moving behavior and querying pattern are stable and known in advance, as assumed in most related work, since both parameters $\alpha$ and $\beta$ depend on these factors. We need to adjust the system in an attempt to reach out for the optimal safe range $r^{*}$, despite the unstable and unknown object movement and query patterns.

\section{Adaptive Safe Range Algorithms}

With unknown system parameters and changing system conditions, one feasible way to strive for optimal system performance is the adaptive adjustment approach. We design two adaptive algorithms for setting the safe range towards minimizing total system cost. The basic idea behind the algorithms is from an observation on the intuitive relationship between the two kinds of costs and safe range $r$. Intuitively, larger $r$ leads to lower tracking cost but higher querying cost and vice versa. Whenever a location update occurs, it is a signal that the safe range should be larger and the value of $r$ should be increased for the next updating ac- signal that the safe range is too large for the precision constraint of the existing query and $r$ should be decreased. This approach adapts the value of $r$ according to changing situation. We then need to determine by what amount should $r$ be adjusted. Too large a jump will create a strong ping-pong effect, defeating the purpose of the adaptation. Too small a shift will make it a lengthy process to adapt to a new object movement and querying pattern.

To determine the adjustment, we look at the system property at the optimal performance point and maneuver the adjustment based on the existing deviation from the optimal property. We observe that the ratio between $\phi(r)$ and $\rho(r)$ is a constant at the optimal safe range $r^{*}$. When $\phi(r) / \rho(r)=2\left(C_{u} / C_{p}\right), r=r^{*}=2\left(C_{u} / C_{p}\right)(\sqrt[3]{\alpha / \beta})$. We thus define $\delta^{*}=2\left(C_{u} / C_{p}\right)$, and the optimal safe range $r^{*}$ is resulted when $\delta=\delta^{*}$. Our problem then reduces to adjusting the safe range $r$ so that $\delta / \delta^{*}=1$. The value of $r$ is adjusted by an amount of $\tau$, a tunable parameter.

Figures 5 and 6 present two adaptive algorithms for setting $r$ at the server. The meaning of symbols used can be found in Table 1. Both algorithms attempt to adjust the system parameters in order to make the ratio of the observed query probe rate and location update rate equal to $\delta^{*}$.

History-tracking Algorithm (HA) keeps track of the location update and query probe rates, in order to compute $\delta$ and make the ratio $\delta / \delta^{*}$ close to 1 by adjusting $r$. Since larger $r$ leads to higher query probe and lower location update rate and hence larger $\delta, \mathrm{HA}$ decreases $r$ when the ratio $\delta / \delta^{*}$ is larger than the value in optimal condition, i.e., 1 . Note that since the value of $\delta$ can change dynamically, HA needs to track changes in object movement and query patterns, by means of an exponentially weighted moving average of the metric, with an adjustable weight $\omega$. To avoid the ping-pong effect, a change is initiated only when the ratio is beyond a certain threshold $\epsilon$ from the target value of 1 .

Non-History-tracking Algorithm (NHA) only makes use of the local property of location update and query probing observed at the server under normal operation, without attempting to track for the object movement pattern, query pattern and query precision, nor storing their history. As with HA, NHA attempts to make the ratio $\delta / \delta^{*}$ close to 1 , but by means of probabilistic approach. To explain the algorithm, let us examine the simplest case when $\delta^{*}=1$. To make the ratio $\delta / \delta^{*}=1$, the system should keep $\delta=1$, i.e., query probe rate should be equal to location update rate. To balance the likelihood of the two types of updates, NHA would decrease or increase $r$ on a query probe or object update in order to reach for the optimal setting. If $\delta^{*}>1$, a larger $r$ which leads to a higher query probe rate and smaller object update rate is preferred. Therefore, NHA would still increase $r$ on a location update but would just decrease $r$ with a probability $1 / \delta^{*}$ on a query probe. Conversely, if 
$r$ on every location update.

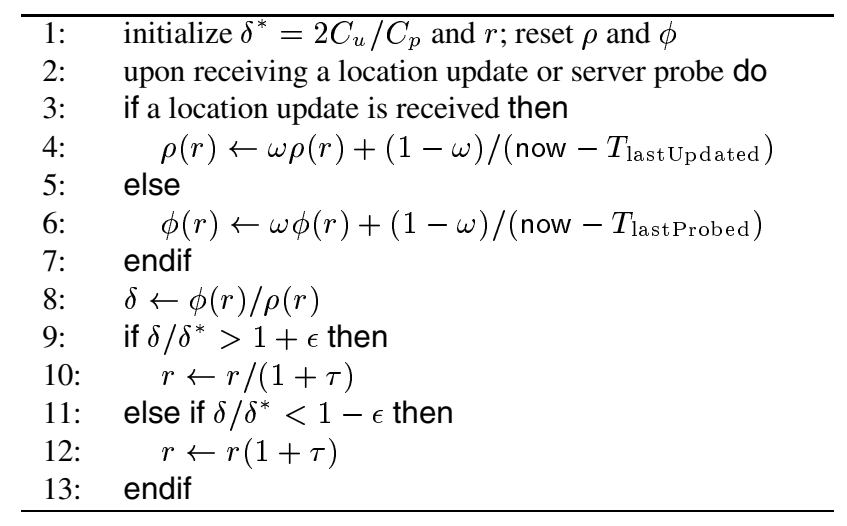

Figure 5. HA at server

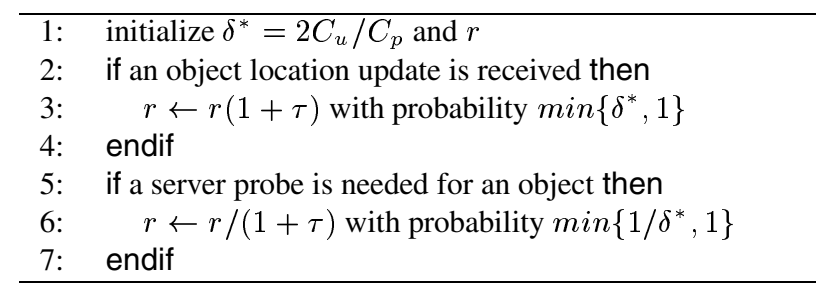

Figure 6. NHA at server

\section{Performance Evaluation}

We conduct a number of experiments to evaluate the performance of our adaptive algorithms. Each experiment models the movement of the moving objects for 1000 time units. The spatial domain of interest is a square-shaped region of size 500 by 500 . Two mobility models are used in the simulation, namely, Random Walk and Random Waypoint [5]. Both are well-known for performance evaluation of MOD. The random walk model is suitable to simulate small-scale scenarios, while the random waypoint model fits better to large-scale on-purpose movements. In random walk, all objects move in steps and each moves a distance of $d$ along an arbitrary direction at each step, with a duration of $L$. In random waypoint, each object chooses a random point in the space as its destination and moves to it at a speed randomly selected from the range $\left[0, V_{\max }\right]$; upon arrival or expiration of a constant movement period randomly picked from the range $\left[0, T_{\max }\right]$, it chooses a new destination and repeats the same process. Queries arrive with rate $\lambda$ and maximum precision constraint $p_{\max }$. We assume that the location update cost per object is 1 and the server probe cost is 2, with one server paging message and one object reporting message. Thus, $\delta^{*}=2 C_{u} / C_{q}$ would be 1 . Note that the actual value of $\delta^{*}$ depends on the semantics of the particular application system and may vary. However, its choice has no particular effect to the algorithm performance, as long as the value can be estimated accurately. Table 2 summarizes the parameters used in our experiments.

\begin{tabular}{|c|c|c|}
$L$ (in second) & 1 & 1 \\
\hline$d$ (in meter) & 1 (slow), 5 (moderate), 15 (fast) & 5 \\
\hline$\lambda$ & $1-10 s^{-1}$ & 1 \\
\hline$V_{\max }$ & 15 & 15 \\
\hline$T_{\max }$ & 2 & 2 \\
\hline$p_{\max }$ & $10-100$ & 50 \\
\hline$\delta^{*}$ & 1 & 1 \\
\hline
\end{tabular}

Table 2. Simulation parameters setting

\subsection{Experiment \#1}

Our first set of simulation experiments is a comprehensive set, conducted to demonstrate that our algorithms indeed achieve optimal performance with appropriate parameter settings. We evaluate using both movement models. For random walk, we consider a set of moving objects with moderate speed. For random waypoint, we take the maximum velocity to be 15 and maximum stop time 2 time units. We simulate a querying workload with query arrival rate of 1 and a maximum precision constraint of 50. First, we run simulation to establish the correctness of our assumption for the relationship among $r$ and $\phi(r)$ and $\rho(r)$, i.e., to show that generally $\rho(r)$ and $\phi(r)$ are proportional to $1 / r^{2}$ and $r$ respectively. We also want to show that when $\phi(r) / \rho(r)=\delta^{*}$, the total system cost is minimized.

We run the update and querying algorithm with fixed $r$ for each experiment (i.e., we do not adjust $r$ adaptively according to different system situation), but vary $r$ across experiments. We measure the average number of updates and probes per time unit and the results are reported in Figure 7. The measured values for $\phi(r)$ and $\rho(r)$ are found to be proportional to $1 / r^{2}$ and $r$ respectively. From the figure for random walk and random waypoint models, we can verify that the minimal total cost can indeed be attained when $\phi(r) / \rho(r)=\delta^{*}=1$, i.e., at the intersection point of the update rate curve and probe rate curve, where $\rho(r)=\phi(r)$.

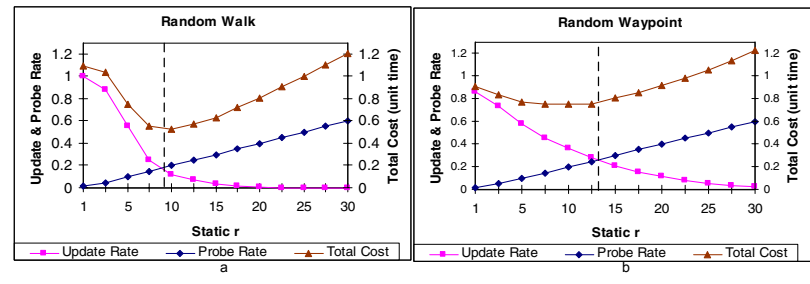

\section{Figure 7. Update/probe rate and cost versus safe range}

Upon confirming the validity of the derived optimal point, we next attempt to validate the claim that our adaptive algorithms can adapt the safe range $r$ towards the optimal value $r^{*}$, i.e., they converge. Both algorithms control the magnitude of the safe range adjustment by means of $\tau$, an important tunable parameter. We vary $\tau$ as well as initial safe range $r^{0}$ in our experiment. After conducting numerous experiments, we could deduce the optimal 
(i.e., the optimal value $r^{*}$ returned by $\mathrm{HA}$ and $\mathrm{NHA}$ ). Figure 8 presents the simulation results for NHA. Under both random walk and random waypoint movement models, it is clear that NHA is unable to yield a best performance when $\tau$ is either too large or too small. This is because a large $\tau$ induces strong fluctuation to the adjustment of $r$, thus missing the optimal. Too small a value of $\tau$ will lead to a very slow convergence for the system to adapt to the optimal $r$. Figure 8 also reveals that unless $\tau$ is too extreme, initial safe range $r^{0}$ brings little impact to the performance. We repeat the experiment to measure the performance of $\mathrm{HA}$ and the results are very similar to those in Figure 8. Table 3 summarizes the best setting for the adjustable parameters and the best safe range $r^{*}$ returned by the algorithms. Compared with the results in Figure 7, the best safe ranges returned from both algorithms are close to the optimal, with at most $5 \%$ difference. The results also indicate that $\mathrm{HA}$ performs better in exerting a lower total system cost.

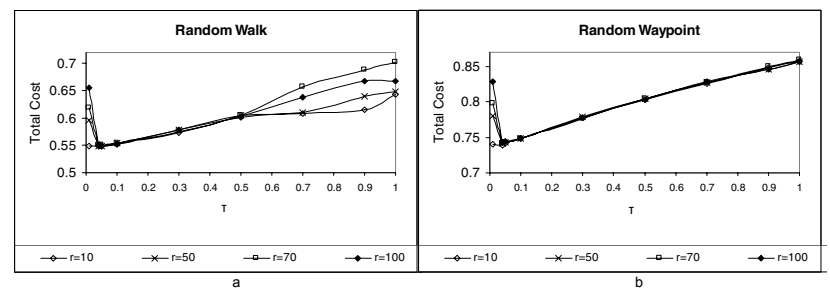

Figure 8. Adaptation of NHA to parameters

\begin{tabular}{|c||c|c||c|c|}
\hline \multicolumn{1}{|c||}{} & \multicolumn{2}{c||}{ NHA } & \multicolumn{2}{c|}{ HA } \\
\hline Parameter & Ran. walk & Ran. waypoint & Ran. walk & Ran. waypoint \\
\hline$\tau$ & 0.04 & 0.04 & 0.04 & 0.04 \\
\hline$r^{0}$ & 50 & 50 & 50 & 50 \\
\hline$\omega$ & - & - & 0.1 & 0.1 \\
\hline$\epsilon$ & - & - & 0.1 & 0.1 \\
\hline$r^{*}$ & 9.064 & 12.255 & 8.318 & 12.134 \\
\hline \hline $\operatorname{cost} \mathcal{C}$ & 0.549 & 0.742 & 0.510 & 0.667 \\
\hline
\end{tabular}

Table 3. Optimal setting for parameters

\subsection{Experiment \#2}

Our second set of experiments aims at evaluating the effect of object movement speed. We employ the random walk movement model, with three movement speeds, i.e., slow, moderate and fast. We set the tunable parameters to their optimal setting according to the experimental results in Experiment \#1. The results are presented in Figure 9. The total system cost increases when moving speed of objects gets faster no matter which algorithm. The reason behind this phenomenon is that faster object movement makes them more easily moving out of their safe region, dictating tight tracking. It is apparent that $\mathrm{HA}$ is more adaptive to the change of speed. This is because NHA uses a fixed pre-determined value of $\delta^{*}$, while $\mathrm{HA}$ attempts to track the changes in object movement pattern with $\delta$. Furthermore, we repeat Experiment \#1 with those varying move- trend, except with minor shifting. Table 4 indicates the best value of $r$ that can be attained by both algorithms and the range of optimal $r$ derived from the static algorithm.

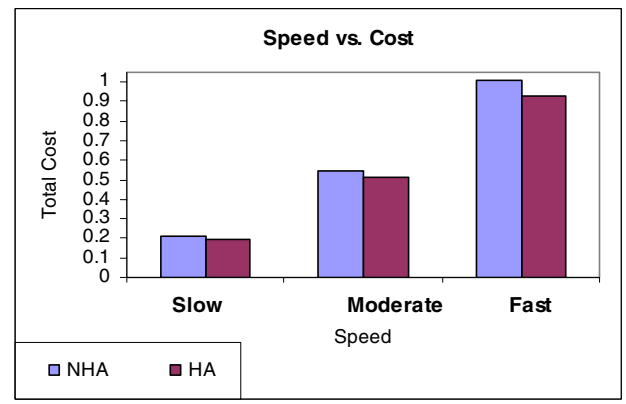

Figure 9. Effect of movement speed

\begin{tabular}{|c||c|c|c|}
\hline Algorithm & slow & moderate & fast \\
\hline NHA & 3.271 & 9.064 & 16.633 \\
\hline HA & 2.997 & 8.318 & 16.900 \\
\hline Algorithm with static $r$ & $3-3.5$ & $8.5-9$ & $16.5-17$ \\
\hline
\end{tabular}

Table 4. Optimal safe range $r$

\subsection{Experiment \#3}

Our third experiment evaluates the effect of the query pattern, with random waypoint movement model. We focus on evaluating the effect on the query arrival rate $\lambda$ and varying query precision $p_{\max }$. The results are depicted in Figure 10. Both algorithms result in higher system cost at higher query rate and smaller maximum precision constraint. This is expected due to a higher degree of querying activities. In both cases, it is clear that HA is performing better with a lower total cost, due to its stronger ability to adapt to changes, compared with NHA with a precomputed $\delta$. There is a performance tradeoff between the two algorithms. NHA requires less computational power at the server because no history tacking is needed while HA can adapt to the changing situation more quickly, yielding better performance at the expense of higher computational cost.

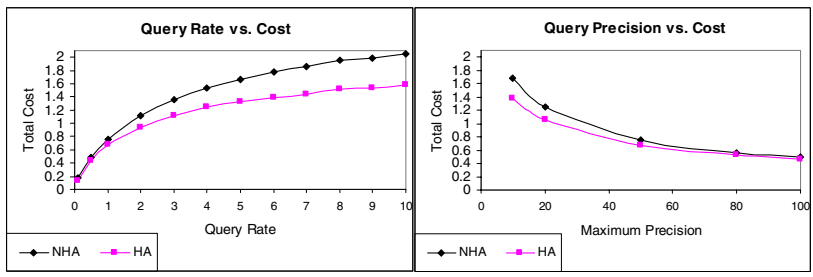

Figure 10. Effect of query pattern

\subsection{Experiment \#4}

Our last set of experiments is conducted to evaluate the robustness of the adaptive algorithms. The aim is to find out how fast our algorithms converge to their best safe range setting when system conditions such as object movement pattern, query arrival rate and query precision requirement 
models to generate two sets of simulation data to model changing movement pattern and query pattern respectively. Dataset1 slows down random walk moving objects after every one-third of the simulation period. Dataset2 is based on the random waypoint model with default maximum speed, but the query pattern is changed from very infrequent and loose precision constraint to frequent and tight precision constraint every one-third of the simulation period. To visualize the changing condition, the query pattern also changes suddenly every one-third of the period. We run both algorithms on the two datasets. The results are shown in Figure 11, with HA1 standing for running HA on Dataset1 and so on. Both adaptive algorithms can converge to stable safe range after some adaptation period. HA exhibits a slightly better performance with faster convergence, especially under changing system conditions. This is quite expected because HA keeps monitoring object update and server probe rates and adapts faster according to a more accurate value of $\delta$, while NHA makes use of a precomputed $\delta^{*}$ no matter how the current condition changes.

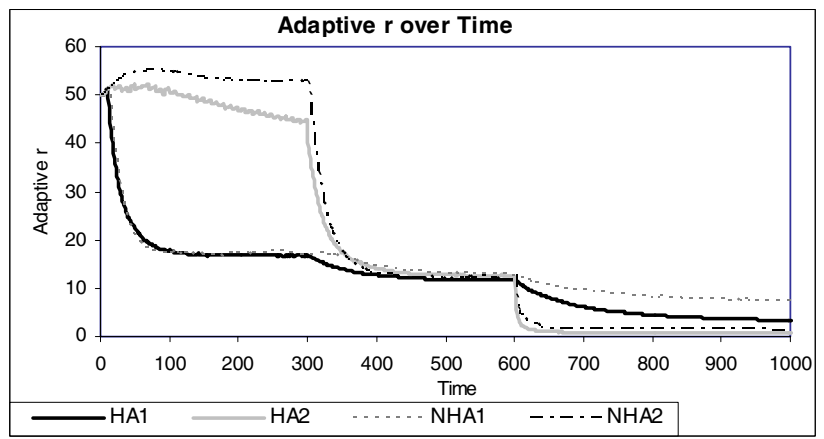

Figure 11. Convergence of algorithms

\section{Conclusion}

In this paper, we considered the location update problem with bounded level of inconsistency, by means of the safe range. We derived the optimal setting of the safe range for the location information based on object movement pattern and query pattern in an ideal known scenario. We then presented two adaptive algorithms for adjusting the safe range that controls the deviation of cached location of moving objects adaptively to achieve the best performance as movement pattern, query precision requirement or system workload vary, as in real applications. Comprehensive simulation studies demonstrate that our algorithms can achieve optimal system performance in a robust manner.

We have so far assumed that the queries are looking for the location of objects. We have not considered other types of useful queries, such as range queries, continuous queries, nearest neighbor queries, etc. As future work, we plan to explore the possibility of applying the adaptive approach to deal with different types of queries in MOD. As the relationship between the safe range and different types of queries order to handle more complex and interesting queries, such as range queries or nearest surrounder queries [8].

\section{References}

[1] A. Bar-Noy, I. Kessler, and M. Sidi. Mobile users: To update or not to update? ACM-Baltzer Journal of Wireless Networks, 1(2):175-185, 1995.

[2] R. Barga and C. Pu. Accessing imprecise data: An approach based on intervals. IEEE Data Engineering Bulletin, 16(2):12-15, 1993.

[3] B. Chan, A. Si, and H. Leong. A framework for cache management for mobile databases: design and evaluation. Journal of Distributed and Parallel Databases, 10(1):23-57, 2001.

[4] M. Franklin and M. Carey. Client-server caching revisited. In Proc. of International Workshop on Distributed Object Management, 1992.

[5] D. Johnson and D. Maltz. Dynamic source routing in ad hoc wireless networks. In Mobile Computing, Edited by Imielinski and Korth, Kluwer Academic Publishers, 1996.

[6] G. Lam, H. Leong, and S. Chan. GBL: Group-based location updating in mobile wireless environment. In Proc. of International Conference on Database Systems for Advanced Applications, pages 762-774, 2004.

[7] K. Lam, O. Ulusoy, T. Lee, E. Chan, and G. Li. An efficient method for generating location updates for processing of location-dependent continuous queries. In Proc. of International Conference on Database Systems for Advanced Applications, pages 218-225, 2001.

[8] K. Lee, W. Lee, and H. Leong. Nearest surrounder queries. In Proc. of International Conference on Data Engineering, 2006.

[9] C. Olston, B. Loo, and J. Widom. Adaptive precision setting for cached approximate values. In Proc. of SIGMOD Conference, 2001.

[10] C. Olston and J. Widom. Offering a precision performance tradeoff for aggregation queries over replicated data. In Proc. of International Conference on Very LargeData Bases, pages 144-155, 2000.

[11] E. Pitoura and G. Samaras. Locating objects in mobile computing. Knowledge and Data Engineering, 13(4):571-592, 2001.

[12] C. Pu and A. Leff. Epsilon-serializability. Technical report, Columbia University, Computer Science Department, 1990.

[13] O. Wolfson, S. Jajodia, and Y. Huang. An adaptive data replication algorithm. ACM Transactions on Database Systems, 22(2):255-314, 1997.

[14] O. Wolfson, P. Sistla, S. Chamberlain, and Y. Yesha. Updating and querying databases that track mobile units. Distributed and Parallel Databases, 7(3):257-387, 1999.

[15] O. Wolfson and H. Yin. Accuracy and resource consumption in tracking moving object. In SSTD, pages 325-343, 2003.

[16] M. Wong, D. Agrawal, and H. Mak. Bounded inconsistency for type-specific concurrency control. Journal of Distributed and Parallel Databases, 5(1):31-75, 1997.

[17] H. Yu and A. Vahdat. Efficient numerical error bounding for replicated network services. In Proc. of International Conference on Very Large Data Bases, pages 123-133, 2000.

[18] J. Zhou, H. Leong, Q. Lu, and K. Lee. Aqua: An adaptive query-aware location updating scheme for mobile objects. In Proc. of International Conference on Database Systems for Advanced Applications, pages 612-624, 2005. 